Journal Club

Editor's Note: These short, critical reviews of recent papers in the Journal, written exclusively by graduate students or postdoctoral fellows, are intended to summarize the important findings of the paper and provide additional insight and commentary. For more information on the format and purpose of the Journal Club, please see http://www.jneurosci.org/misc/ifa_features.shtml.

\title{
Dendritic Spikes Provide a Mechanism for Hippocampal Replay and Sharp-Wave/Ripple Generation
}

\author{
이 Jake Jordan \\ Biology Department, Queens College, the City University of New York, Flushing, New York 11367, and Biology PhD Program, the Graduate Center, the City \\ University of New York, New York 10016 \\ Review of Jahnke et al.
}

The hippocampus, located in the medial temporal lobe, is essential for the formation of new declarative memories (Scoville and Milner, 1957). In areas CA3 and CA1, recurrent network connectivity allows for the strengthening of synapses between coactive neurons, acting as a mechanism for the learning and storage of new memories. In awake behaving animals, cholinergic modulation suppresses the activity of recurrent hippocampal connections and gives priority to the incoming sensory stream from the entorhinal cortex, which then is encoded into the short-term store of the hippocampus (for review, see Gupta and Hasselmo, 2014). Recall can occur at a later time, by reactivation of the same neurons active during encoding by use of a retrieval cue (Garner et al., 2012; Liu et al., 2012).

How are memories that are formed during alert behavior consolidated from the hippocampus into the long-term store of the cortex? The "two-step" hypothesis of memory consolidation suggests that through the replay of memories, the hippocampus can sufficiently potentiate cortical synapses for the long-term storage of new experiences (Marr, 1971; Buzsáki, 1989). Marr (1971) suggested that this

Received Feb. 9, 2016; revised March 6, 2016; accepted March 8, 2016. The authors declare no competing financial interests.

Correspondence should be addressed to Jake Jordan, Queens College,

272 Razran Hall, 65-30 Kissena Boulevard, Flushing, NY 11367. E-mail: jake.jordan@qc.cuny.edu.

DOI:10.1523/JNEUROSCI.0442-16.2016

Copyright $\odot 2016$ the authors $\quad 0270-6474 / 16 / 364152-03 \$ 15.00 / 0$ could only occur in the absence of significant sensory processing, such as during sleep. Indeed, during sleep, ensemble recordings of single neurons have detected sequential reactivation of overlapping place cells that resemble recordings taken during encoding of a novel environment (Wilson and McNaughton, 1994). In addition, local field potential recordings in the hippocampus during states of low activity demonstrate a distinct physiological signature called the sharp-wave/ripple (SPW/R) complex, which is characterized by heightened overall activity (sharp waves) coupled with fast oscillations of inhibitory and excitatory activity (ripples). These events display temporal dynamics, which suggest they may be important for synaptic modification in cortical targets whose activity increases after SPW/Rs as would be predicted by the "two-step" hypothesis (Markram et al., 1997). Consistent with this hypothesis, enhancing or suppressing SPW/Rs enhances and impairs memory consolidation, respectively (Girardeau et al., 2009; Barnes and Wilson, 2014).

How such activity is generated in the absence of sensation is unclear. However, it is known that in the absence of cholinergic modulation, activity at recurrent synapses within the hippocampus is dominant (Gupta and Hasselmo, 2014), which may be due to highly synchronous input from the cortex. The relationships between learning, replay, and SPW/Rs is also unknown, although several hypotheses have been offered, including a possible involvement of dendritic amplification of input. In a recent computational study, Jahnke et al. (2015) incorporated nonlinear dendrites, whose membrane potentials spike after input has reached a certain threshold, into recurrently connected networks resembling those of the hippocampal subregions CA1 and CA3 to examine how they might contribute to SPW/R and replay generation.

To understand how SPW/Rs and replay are related to the consolidation of recent memories into the cortical store, Jahnke et al. (2015) trained a recurrent artificial neural network to learn a virtual sequence of spatial locations. This network consisted of 2500 excitatory nodes and 250 inhibitory nodes. Place fields were randomly assigned, as were initial synaptic weights. Importantly, dendritic spikes occurred at recurrent synapses when input into these dendrites exceeded a certain threshold. A current pulse would then be sent from the dendrite to the soma, resulting in a greater depolarization than would be provided from summated input (Jahnke et al., 2015, their Fig. 1).

They trained this network by delivering current pulses to cells when the virtual animal traversed the cells' randomly assigned place fields. To mimic hippocampal physiology, these current pulses were locked into a background signal, meant to play the role of the hippocampal theta oscillation. Learning in this network was based on a spike-timing-dependent plas- 
ticity (STDP) rule. Though initially randomly distributed, synaptic weights could be modified such that synapses exhibiting causal spikes (ie, postsynaptic spikes occurring soon after presynaptic spikes) were potentiated, whereas anticausal activity (when postsynaptic spikes preceded presynaptic spikes) was depotentiated. This resulted in a feedforward network structure (Jahnke et al., 2015, their Fig. $4 D, E$ ), meaning that neuronal activity was sequential based on the individual cells' place fields along the track. However, after a high number of trials this network demonstrated pathological "overlearning" (Jahnke et al., 2015, their Fig. 5) and was thus modified so that potentiation and depotentiation occurred only when causal and anticausal activity occurred within a specific timeframe.

After learning, the authors changed the current pulses delivered to the network to less resemble active learning and to more resemble cortical input to the hippocampus during rest, immediately preceding SPW/Rs. This input was highly synchronized and delivered to only a subset of the network with nearby place-field centers. This resulted in a cascade of activity across the network. To determine whether this activity resembled replay of the previous experience, the order of individual spike pairs during replay was examined in relation to the ordering during learning. The total number of "false" spike pairs, in which the two nodes reversed spiking order between learning and replay, was subtracted from the total number of "true" spike pairs in which the order was preserved. This was normalized by the total number of spike pairs examined, such that the matching index $(I)$ between learning and replay was as follows:

\section{$I=($ True Spike Pairs \\ - False Spike Pairs)/(True Spike

$$
\text { Pairs + False Spike Pairs) }
$$

After just five runs along the virtual track, synchronous stimulation could elicit replay that had a matching index significantly $>0$ (Jahnke et al., 2015, their Fig. 8, compare $B, C)$. With more trials, the matching index grew (Jahnke et al., 2015, their Fig. 8B). As the number of trials increased, the number of stimulated neurons needed to achieve the optimal matching index became smaller (Jahnke et al., 2015, their Fig. 8 B). Furthermore, nonspecific stimulation of a subset of this network (rather than a subset with neighboring place cells), also resulted in a positive matching index (Jahnke et al., 2015, their Fig. 10).

During replay, nodes that received synchronous stimulation stimulated an even greater number of postsynaptic nodes to spike. This second pulse elicited an even greater third pulse, as more and more neurons become excited. This increased overall activity, resembling the sharp wave events, contained within it complementary oscillations of excitatory and inhibitory activity (Jahnke et al., 2015, their Fig. 7C-F), which both prevents epileptic activity and resembles ripple events. Importantly, this replay occurred at a frequency $(\sim 200 \mathrm{~Hz})$ similar to the frequency found experimentally $(150-250 \mathrm{~Hz})$. Furthermore, this replay only occurred when the incoming stimulant was sufficiently synchronized across the network. Thus, replay did not occur during the learning of the virtual track.

One of the essential tasks that the hippocampus must carry out is the separation of multiple memory traces. The network in this study was able to learn and replay multiple virtual tracks. When replay was elicited after learning multiple tracks, only synchronous stimulation of neurons involved in a particular sequence resulted in a replay matching index that was substantially $>0$ (Jahnke et al., 2015, their Fig. 11A). When inducing replay, stimulation of a subset of neurons that encoded track 3, for instance, resulted in positive $I$ values when the spike sequences were compared with encoding of track 3 , but resulted in $I$ values closer to 0 compared with the encoding activity of track 2 (Jahnke et al., 2015, their Fig. 11A).

In summary, this computational model showed that after undergoing STDP-based synaptic modification during learning, feedforward structures within a recurrent network form, storing the experience. Upon synchronized stimulation of a subset of a stored memory trace, dendritic spikes trigger a replay of activity patterns present at learning. This replay is characterized by an overall increase in network activity coupled with a high-frequency oscillation of inhibitory and excitatory activity, resembling the hippocampal SPW/Rs. Thus, this model unifies learning, replay, and SPW/Rs. From this model, the authors make three predictions: (1) dendritic spikes are present at recurrent synapses in both CA3 and CA1; (2) these dendritic spikes generate the action potentials involved in replay and SPW/Rs; and (3) if dendritic spikes can be selectively abolished, replay and SPW/R generation will be impaired.
In addition, this study has provided clues about what the cortical input the hippocampus that induces SPW/R and replay activity must resemble. This input has to be highly synchronous and must target a subset of a particular memory trace in order for the replay to match that particular experience. Hippocampal memory traces can remain in the hippocampus for days and perhaps weeks (Denny et al., 2014). Thus, at any one time, the hippocampus stores many individual traces. It will be important to determine whether cortically driven replay of certain traces is preferentially generated over other, perhaps less adaptive, memory traces. This may work as a gating mechanism to determine which memories become stabilized and consolidated into the long-term store, and which memories are subsequently forgotten. It has been suggested that dopaminergic hippocampal modulation during novel or rewarding experiences may enhance synaptic modification in the hippocampus, increase the probability that these experiences will be replayed and promote the consolidation of these experiences (Lisman and Grace, 2005; Atherton et al., 2015), thus acting as a gating mechanism.

The role of replay and SPW/Rs in the consolidation of specific memory traces during low-activity has yet to be investigated. What may be of use to investigators are recently developed genetic mouse models that can tag an encoding population with fluorescent reporters (Liu et al., 2012; Denny et al., 2014), designer receptors exclusively activated by designer drugs (Garner et al., 2012), channelrhodopsins (Liu et al., 2012; Denny et al., 2014), or other proteins of interest. For instance, expression of calcium indicators in these populations may be combined with calcium imaging in dorsal CA1 and cortex to understand how replay and SPW/Rs play a role in clearing these traces from the hippocampus or consolidating them into the long-term cortical store.

\section{References}

Atherton LA, Dupret D, Mellor JR (2015) Memory trace replay: the shaping of memory consolidation by neuromodulation. Trends Neurosci 38:560-570. CrossRef Medline

Barnes DC, Wilson DA (2014) Slow-wave sleep-imposed replay modulates both strength and precision of memory. J Neurosci 34:5134-5142. CrossRef Medline

Buzsáki G (1989) Two-stage model of memory trace formation: a role for "noisy" brain states. Neuroscience 31:551-570. CrossRef Medline 
Denny CA, Kheirbek MA, Alba EL, Tanaka KF, Brachman RA, Laughman KB, Tomm NK, Turi GF, Losonczy A, Hen R (2014) Hippocampal memory traces are differentially modulated by experience, time, and adult neurogenesis. Neuron 83:189-201. CrossRef Medline

Garner AR, Rowland DC, Hwang SY, Baumgaertel K, Roth BL, Kentros C, Mayford M (2012) Generation of a synthetic memory trace. Science 335:1513-1516. CrossRef Medline

Girardeau G, Benchenane K, Wiener SI, Buzsáki G, Zugaro MB (2009) Selective suppression of hippocampal ripples impairs spatial memory. Nat Neurosci 12:1222-1223. CrossRef Medline
Gupta K, Hasselmo M (2014) Modulatory influences on the hippocampus and entorhinal cortex. In: Space, time, and memory in the hippocampal formation, Chap 7, pp 153-189. Heidelberg: Springer.

Jahnke S, Timme M, Memmesheimer RM (2015) A unified dynamic model for learning and replay, and sharp-wave/ripples. J Neurosci 35: 16236-16258. CrossRef Medline

Lisman JE, Grace AA (2005) The hippocampalVTA loop: controlling the entry of information into long-term memory. Neuron 46: 703-713. CrossRef Medline

Liu X, Ramirez S, Pang PT, Puryear CB, Govindarajan A, Deisseroth K, Tonegawa S (2012) Optogenetic stimulation of a hippocampal engram activates fear memory recall. Nature 484:381-385. CrossRef Medline

Markram H, Lübke J, Frotscher M, Sakmann B (1997) Regulation of synaptic efficacy by coincidence of postsynaptic APs und EPSPs. Science 275:213-215. CrossRef Medline

Marr D (1971) Simple memory: a theory for archicortex. Philos Trans R Soc Lond B Biol Sci 262:23-81. CrossRef Medline

Scoville WB, Milner B (1957) Loss of recent memory after bilateral hippocampal lesions. J Neurol Neurosurg Psychiatry 20:11-21. CrossRef Medline

Wilson MA, McNaughton BL (1994) Reactivation of hippocampal ensemble memories during sleep. Science 265:676-679. CrossRef Medline 